Aims. To ensure that service users in in-patient secure services have prolactin, vitamin $\mathrm{D}$ and $\mathrm{HbA} 1 \mathrm{c}$ monitoring as per current best practice guidance.

Background. Service users prescribed antipsychotic medication are at risk of developing raised prolactin levels and metabolic syndrome. In both sexes, long-standing hyperprolactinaemia can lead to low bone mineral density with an increased risk of developing osteoporosis.

In recent years there has been increasing controversy on the increase in Vitamin D monitoring despite the poor evidence for complications from vitamin $\mathrm{D}$ deficiency in adults. Not undertaking this test in the absence of symptoms will potentially reduce anxiety for service users could save $£ 17$ per test and $£ 50$ for a 12-week course of Vitamin D supplementation. Local and national guidance indicate Vitamin D monitoring should only be done in symptomatic people.

Method. Fifty-five service users in the five in-patient wards had their electronic records and pathology results reviewed over a oneyear period. All service users were expected to have a minimum of an annual $\mathrm{HbAlc}$ and prolactin level but to only have vitamin D monitoring if symptomatic for deficiency.

Result. Although $100 \%$ of service users in MSU were tested, vitamin D testing was consistently undertaken without documented clinical evidence of deficiency. The ranges across all units were: prolactin $(72-1384 \mathrm{mU} / \mathrm{L}), \mathrm{HbAlc}(30-90 \mathrm{mmol} / \mathrm{mol})$ and vitamin $\mathrm{D}(15-124 \mathrm{nmol} / \mathrm{L})$. Local reference ranges are prolactin (53- 360mU/L), HbAlc (<48 mmol/mol) and Vitamin D (50$120 \mathrm{nmol} / \mathrm{L})$.

Prolactin levels were highest on the male medium secure wards.

The other two units had significantly less testing with prolactin and $\mathrm{HbAlc}$ levels being the least measured (18\% of service users on male LSU and $23 \%$ on the female ward respectively). Vitamin D testing on these two wards were $38 \%$ on the female ward and $18 \%$ on the male ward for both tests.

Conclusion. Northside House has a dedicated physical health team and this is likely to explain its $100 \%$ score. However, vitamin $\mathrm{D}$ testing was being undertaking automatically rather than based on symptoms.

The recommendation is to add prolactin and $\mathrm{HbAlc}$ to the physical screens done before CPA meetings for all service users prescribed an antipsychotic but to stop Vitamin D testing in the absence of clinical symptoms of vitamin D deficiency.

Advanced nurse practitioners- the missing link on old age pyschiatry inpatient wards?

Grace Lydia Goss ${ }^{1 *}$, Priya Gowda ${ }^{2}$ and Danika Rafferty ${ }^{2}$

${ }^{1}$ Aneurin Bevan University Health Board and ${ }^{2}$ Cwm Taf Morgannwg University Health Board

${ }^{*}$ Corresponding author.

doi: 10.1192/bjo.2021.869

Aims. 1. This project aimed to review the medical cover available to an Old Age Psychiatry inpatient ward.

2. To discuss with ward staff their view for potential improvements and areas of clinical development.

3. To review the potential of a Full Time Nurse Practitioner role on the ward.

One junior doctor (CT1 or equivalent) was allocated to cover the ward whilst balancing their other training needs and clinical commitments. The inpatient ward was based in a community hospital with no onsite medical team. The patients mostly had complex medical needs and multiple comorbidities.
Method. The Junior Doctor's timetable and the time allocated to the ward was reviewed. Questionnaires were conducted with nursing staff to assess their views on the support of physical health cover. The patient notes were analysed for the time taken to review patients after falls over a one month period.

Result. There were 14.5 hours allocated to ward cover. An additional 4 hours was provided by another visiting junior doctor totalling 18.5 hours per week- $11 \%$ of the time. This figure does not account for annual leave, on call commitments or study days whereby there was no additional cover.

A short survey completed by ward staff showed- $(1=$ Very Poor/Difficult 5 = Excellent/Easy)

- They rated medical cover of physical health needs on ward 7 as 1.3 .

- They found contacting a Doctor to discuss a physical problem as 1.7- with particular concern for $\mathrm{OOH}$.

- It was rated to be extremely difficult for a same day review of physical health problems- 1.7

- It was rated extremely difficult to get a physical review following a fall on ward 7- 1.4

- Continuity of care for the patients on ward 7 was rated as 1.6.

The patient case files reviewed over a one month period showed $\mathrm{x} 8$ falls. These took on average 14 hours before having a review. Conclusion. Medical cover for the old age psychiatry inpatient ward was inconsistent and a challenge for a single trainee to manage alongside their other clinical commitments and training needs. A case was proposed to management with an SBAR for a Full Time Advanced Nurse Practitioner which has been approved. This role should provide patients with appropriate cover of their physical health needs. It will allow the junior doctor to work alongside them on the ward supporting each other to provide optimal care for the inpatients.

\section{Audit on venous thromboembolism risk assessment in mental health inpatient wards}

\section{Rahul Malhotra}

Bryn Y Neuadd Hospital, Betsi Cadwaladr University Health Board

doi: 10.1192/bjo.2021.870

Aims. From May 2015 NHS organisations in Wales are expected to report the number of VTE cases associated with hospital admissions which are possible hospital acquired thrombosis (HAT) per calendar month. NICE Quality Standards (QS3) recommend that All patients, on admission, receive an assessment of VTE and bleeding risk using the clinical risk assessment criteria described in the national tool.

Background. VTE is a condition in which a blood clot (thrombus) forms in a vein, most commonly in the deep veins of the legs, known as a deep vein thrombosis (DVT). The thrombus can dislodge from its original site and travel in the blood (embolism). If it becomes lodged in the lungs, a condition known as a pulmonary embolism (PE) arises and can cause sudden death. Hospital acquired thrombosis is avoidable and unfortunately kills patients under our care. Method. Collected data using a standardised form for 131 patients from 3 inpatient mental health units on documentation of a VTE risk assessment in the inpatient notes. For those patients who had a documented risk assessment, further data were collected on documentation of contraindications, eisk factors, sign and date of prescription and the appropriateness of prescribing. Conclusion. $8 \%$ of patients from one mental health unit $(n=48)$ had a documented risk assessment in the notes. The subsections of 
documented risk assessment including contraindications, risk factors, sign and date of prescriptions and appropriateness of prescribing were complete at $100 \%$. No patients from the other 2 mental health units $(n=39,44)$ had a risk assessment documented in the notes.

Recommendations: All adult inpatients in Mental Health units must receive a venous thrombo-embolism risk assessment. This must be documented on the Inpatient Medication Chart. Consider adding a risk assessment checklist tool mapped from the Department of Health guidelines into the Mental Health Inpatient Clerking in pro-forma.

\section{Service evaluation of long acting buprenorphine subcutaneous injection (BUVIDAL) in the west Lothian community addictions service}

Amy Martin

St. John's Hospital

doi: 10.1192/bjo.2021.871

Aims. 1. To establish if long acting buprenorphine subcutaneous injection retains patients in treatment.

2. To obtain the patient opinion of long acting buprenorphine subcutaneous injection and ascertain if it improved other aspects of their life for example relationships and employment.

Method. Information was gathered from TRAK, the patient record recording system, and Illy, the prescribing system. This allowed data to be gathered on previous opiate substitute treatments and when the patient was commenced on the long acting buprenorphine injection. A patient questionnaire was used to obtain qualitative data on the patient's view of this treatment option.

Result. West Lothian Community Addictions Service starting offering long acting buprenorphine injection as a treatment option in March 2020. Since then there has been a consistent demand from patients to be commenced on this treatment. On 31st January $202139 / 53$ (73.6\%) of patients who had been commenced on long acting buprenorphine for 6 months had been retained on this treatment. Moreover, 3 patients were lost to treatment due to transfer to Her Majesty's Prison. Patients who were commenced on this treatment option were both new to treatment and those who had previously been difficult to retain on methadone or sublingual buprenorphine. The questionnaire supported the antidotal feedback that patients found this treatment option to be hugely beneficial.

Conclusion. Long acting buprenorphine injection has been well tolerated by patients and there has been a clear demand for this treatment option from patients accessing the service. It appears that the clarity of mind, that is associated with buprenorphine, has not been a barrier to retention in treatment. We have found the retention rate of the patients on this treatment option has been higher than the median 6 month retention for either methadone or buprenorphine, compared to a recent systematic review. In addition, it has helped patients consider employment, improve relationships and maintain a level of stability that they may not have previously achieved on either methadone or sublingual buprenorphine.

\section{Electroconvulsive therapy (ECT) and schizophrenia}

Rhys Masin ${ }^{1 \star}$ and George Kirov ${ }^{2}$

${ }^{1}$ Cardiff University School of Medicine and ${ }^{2}$ ECT Department, Cardiff \& Vale University Health Board, Cardiff University School of Medicine

${ }^{\star}$ Corresponding author.

doi: 10.1192/bjo.2021.872
Aims. An evaluation of the benefits of ECT in patients with schizophrenia who received ECT in Cardiff \& Vale UHB, in order to:

- Investigate the effectiveness of ECT as a treatment for schizophrenia

- Inform future clinical practice

- Identify clear outcome measures for use in future research

Background. Schizophrenia is a severe and debilitating mental illness, for which pharmacological treatment is often ineffective. ECT is seldom used for schizophrenia, despite encouraging evidence for its efficacy. Current guidance in the UK is inconsistent, as RCPsych contemplates the use of ECT in schizophrenia in certain cases, while NICE does not. This confusion warrants the need for further evaluation of ECT as a treatment for schizophrenia. Method. Eight suitable patients were identified, and a retrospective chart review was conducted in relation to the following outcomes:

- What was the main indication for ECT, and was the issue resolved

- Change in clinical rating scales

- Concordance with medication before and after treatment

- Length of hospital stay before and after treatment, over one year

- Mental Health Act status after treatment for those treated on section

- Was the level of observation reduced following treatment

Result. Initial indication for treatment was completely resolved in seven out of eight cases. All patients improved in overall symptomatic score (mean improvement $=59.5 \%$ ). Five patients $(62.5 \%)$ improved above the threshold of clinically significant response. At the commencement of treatment, three (37.5\%) patients were refusing all medication, three (37.5\%) had poor concordance and two (25\%) were fully concordant. At treatment endpoint, all were fully concordant. Average length of hospital stay remained unchanged: 30 weeks during the year before ECT, and 33 weeks during the year after ECT. Of six patients treated under Section 3, four (66.7\%) had their section lifted within six months. Observation level was reduced in all cases that had been placed under continuous observation.

Conclusion. ECT improved all outcomes except admission duration. These results provide support for the consideration of ECT as a meaningful treatment option for schizophrenia.

\section{PERCEIVE - Patterning Employment, Race, and} Clinical Experience In Violence against Employees

Ben McClure ${ }^{1 *}$ and Keith Reid ${ }^{2}$

${ }^{1}$ Hopewood Park Hospital, Cumbria, Northumberland Tyne Wear NHS Foundation Trust and ${ }^{2}$ Cumbria, Northumberland Tyne and Wear Foundation Trust, Northumbria University

${ }^{*}$ Corresponding author.

doi: 10.1192/bjo.2021.873

Aims. PERCEIVE is a service evaluation counting whether nurse demographics correlate with reported subjection to violence and verbal aggression. The setting was a large mental health, learning disability and neuropsychiatry NHS trust in England. This continues our work to understand correlations reported in the literature between temporary staff and violence.

Method. We consulted the Caldicott, legal, equality \& diversity, teams and gained service evaluation permission SER-19-031 